\title{
DOES MARKOV-MODULATION INCREASE THE RISK?
}

\author{
By SøRen Asmussen, Aalborg University, Denmark, \\ ANDREAS FREY, University of Ulm, Germany, \\ TOMASZ ROLSKI, University of Wroclaw, Poland, \\ VOLKER SCHMIDT, University of Ulm, Germany
}

\begin{abstract}
In this paper we compare ruin functions for two risk processes with respect to stochastic ordering, stop-loss ordering and ordering of adjustment coefficients. The risk processes are as follows: in the Markov-modulated environment and the associated averaged compound Poisson model. In the latter case the arrival rate is obtained by averaging over time the arrival rate in the Markov modulated model and the distribution of the claim size is obtained by averaging the ones over consecutive claim sizes.
\end{abstract}

\section{KEYWORDS}

Risk theory, compound Poisson model, Markovian environment, stochastic ordering, stop-loss ordering, adjustment coefficient, exponential tail ordering, perturbation.

\section{INTRODUCTION}

This paper is concerned with ordering of risks, more precisely with comparing the ruin functions

$$
\psi(u)=\mathbb{P}\left(\inf _{t \geq 0} R(t)<0 \mid R(0)=u\right), \quad \psi^{*}(u)=\mathbb{P}\left(\inf _{t \geq 0} R^{*}(t)<0 \mid R^{*}(0)=u\right)
$$

of two related risk processes $\{R(t)\},\left\{R^{*}(t)\right\}$. This topic is of obvious actuarial interest and has been studied, for example, in Goovaerts et al. (1990), van Heerwarden (1991), Klüppelberg (1993) and Asmussen (1994).

Much of the literature concentrates on assessing whether a given claim size distribution $B$ is more dangerous than another one $B^{*}$ in the sense that

$$
\psi^{*}(u) \leq \psi(u), \text { for every } u \geq 0
$$

when $B, B^{*}$ are the claim size distributions of two standard compound Poisson risk processes $\{R(t)\},\left\{R^{*}(t)\right\}$ with the same arrival intensity $\beta=\beta^{*}$ and the same premium rate $p=p^{*}$; the ordering (1.2) is referred to as stochastic ordering and we write $\psi^{*}<_{\text {so }} \psi$ (more generally, the ordering relations studied in this paper are defined for functions in $\mathscr{R}$, the class of monotone functions defined on $[0, \infty)$, decreas- 
ing to zero at infinity and assuming values between zero and one). In the compound Poisson setting, it is easy to show that if $B$ is stochastically larger than $B^{*}$ (that is, if $\bar{B}^{*}<_{\text {so }} \bar{B}$ where $\bar{B}(x)=1-B(x)$ is the tail), then (1.2) holds. This follows immediately from the fact that in this case one can assume that the trajectories of the risk processes $\{R(t)\},\left\{R^{*}(t)\right\}$ satisfy the inequality $R^{*}(t) \geq R(t)$ for every $t \geq 0$. A somewhat more substantial result which can be found in Daley and Rolski (1984), see also Makowski (1994), states that (1.2) remains true if $\bar{B}^{*}<_{\text {so }} \bar{B}$ is weakened to $\bar{B}^{*}<_{\mathrm{sl}} \bar{B}$ where, for any two functions $\psi^{(1)}, \psi^{(2)} \in \mathscr{R}$, the stop-loss ordering $\psi^{(1)}<_{\mathrm{sl}} \psi^{(2)}$ is defined by

$$
\int_{x}^{\infty} \psi^{(1)}(u) d u \leq \int_{x}^{\infty} \psi^{(2)}(u) d u, \text { for every } x \geq 0 .
$$

This paper concentrates on the study of the role of deviations from the Poisson arrival pattern. Rather than looking at renewal processes which are mathematically nice but hard to motivate practically, our framework is that of Markov-modulated Poisson arrivals, see Janssen (1980), Reinhard (1984), Janssen and Reinhard (1985), Asmussen (1989), Asmussen and Rolski (1991, 1994), Grigelionis (1993) and Asmussen et al. (1994) for some relevant references. Roughly, the model states that there is an underlying Markov process $\{J(t)\}$ with $p<\infty$ states, such that arrivals occur as in a Poisson process with rate $\beta_{i}$ when $J(t)=i$, and that the corresponding claims have distributions $B_{i}$ with means $\mu_{i}$ (the premium rate may also depend on $i$ but by an operational time argument we may and shall assume that it is 1 in all environmental states). The corresponding risk process is denoted by $\{R(t)\}$ in the following. The motivation for this type of modeling is in part descriptive because of the flexibility, allowing in particular to model arrival streams which are more bursty than any renewal process, but in part also that at least in some cases, one can interpret the model in a natural way. E.g. Asmussen $(1989,1994)$ discusses car insurance where the states of $\{J(t)\}$ describe weather conditions. The model of Janssen and Reinhard (1985) is mathematically slightly different but has a similar flavour from the modeling point of view.

If the $\beta_{i}$ and $B_{i}$ do not fluctuate too much around some average values $\beta^{*}, B^{*}$, one can see the model as a perturbation of a classical compound Poisson risk process $\left\{R^{*}(t)\right\}$ with arrival rate $\beta^{*}$ and claim size distribution $B^{*}$. The rigorous definition of $\beta^{*}, B^{*}$ (which also makes sense and is interesting if the Markov-modulation is more clear-cut) is as follows: We assume that $\{J(t)\}$ is irreducible and time-homogeneous with intensity matrix $\Lambda=\left(\Lambda_{j k}\right)$ and stationary initial distribution $\boldsymbol{\pi}=\left(\pi_{1}, \ldots, \pi_{p}\right)$, i.e. $\boldsymbol{\pi} \boldsymbol{\Lambda}=\mathbf{0}$. Then,

$$
\begin{aligned}
& \beta^{*}(x)=\lim _{t \rightarrow \infty} \frac{N(t)}{t}=\sum_{i=1}^{p} \pi_{i} \beta_{i}, \\
& B^{*}(x)=\lim _{n \rightarrow \infty} \frac{1}{n} \sum_{k=1}^{n} \mathbf{1}\left(U_{k} \leq x\right)=\frac{1}{\beta^{*}} \sum_{i=1}^{p} \pi_{i} \beta_{i} B_{i}(x), \quad x \geq 0
\end{aligned}
$$

where $1(D)$ denotes the indicator of the set $D, N(t)$ is the number of arrivals before $t$ for $\{R(t)\}$ and $\mathrm{U}_{1}, \mathrm{U}_{2}, \ldots$ the corresponding claim sizes so that

$$
R(t)=u+t-\sum_{k=1}^{N(t)} U_{k}, \quad S(t)=\sum_{k=1}^{N(t)} U_{k}-t,
$$

where $S(t)=R(0)-R(t)=u-R(t)$ is the claim surplus at time $t$. Similarly, 


$$
R^{*}(t)=u+t-\sum_{k=1}^{N^{*}(t)} U_{k}^{*}, \quad S(t)=\sum_{k=1}^{N^{*}(t)} U_{k}^{*}-t,
$$

where $\left\{N^{*}(t)\right\}$ is Poisson with rate $\beta^{*}$ and $U_{1}^{*}, U_{2}^{*}, \ldots$ are i.i.d. with distribution $B^{*}$. The fact that the averages $\beta^{*}$ and $B^{*}(s)$ are well-defined and a.s. constant follows from the ergodicity of the environmental process $\{J(t)\}$ which is reflected in corresponding ergodicity properties of the Cox process $\{N(t)\}$ and the sequence $\left\{U_{k}\right\}$ of claim sizes, see e.g. Chapter 8 of König and Schmidt (1992). Moreover, the risk processes $\{R(t)\}$ and $\left\{R^{*}(t)\right\}$ have the same safety loading $\eta=\eta^{*}=\left(\sum_{i=1}^{\mathrm{p}} \pi_{\mathrm{i}} \beta_{i} \mu_{i}\right)^{-1}-1$, which is assumed to be positive.

The initial purpose of the present research was to show that it is always the case that $\psi^{*}<_{\text {so }} \psi$ where the ruin functions $\psi, \psi^{*}$ correspond to the Markov-modulated risk process $\{R(t)\}$ and its averaged compound Poisson counterpart $\left\{R^{*}(t)\right\}$, respectively. The conjecture that such a result could be true came in part from numerical studies, in part from the folklore principle that any added stochastic variation increases the risk, and finally in part from queueing theory, where it has been observed repeatedly that Markov modulation increases several queueing characteristics, see e.g. Ross (1978), Rolski (1981, 1989), Chang et al. (1991), Chang and Nelson (1993). In fact, in the present paper we give a partial solution to our original conjecture showing that $\psi^{*}<_{\text {so }} \psi$ holds under an additional monotonicity condition on the Markov-modulated environment, but also counterexamples showing that at least some conditions are needed.

Without loss of generality we can enumerate the $p$ states of the environment such that

$$
\beta_{1} \leq \beta_{2} \leq \ldots \leq \beta_{p} .
$$

The monotonicity conditions which play an important role in our paper are the following: Assume that, for the numbering of environmental states given by (1.6), we have

$$
\bar{B}_{1}<_{\mathrm{so}} \bar{B}_{2}<_{\mathrm{so}} \ldots<_{\mathrm{so}} \bar{B}_{p},
$$

and stochastic monotonicity of the underlying Markov process (cf. Stoyan (1983)) which with finitely many states can be stated as

$$
\sum_{n \geq l} \Lambda_{j n} \leq \sum_{n \geq l} \Lambda_{k n} \text { for all } \mathrm{j}, \mathrm{k}, \mathrm{l} \text { with } \mathrm{j} \leq \mathrm{k} \text {, and } \mathrm{l} \leq \mathrm{j} \text { or } \mathrm{l}>\mathrm{k} .
$$

To avoid trivialities, we also assume that there exist $i \neq j$ such that either $\beta_{i}<\beta_{j}$ or $B_{i} \neq B_{j}$. Occasionally we strengthen (1.7) to

$$
B \equiv B_{i},
$$

i.e. $B_{i}$ does not depend on $i$. Note that the monotonicity condition (1.8) is automatically fulfilled in some simple examples like birth-death processes or $p=2$.

The main result of the paper is the following:

Theorem 1.1 Assume that conditions (1.6), (1.7) and (1.8) hold. Then $\psi^{*}<_{\mathrm{so}} \psi$.

The proof of Theorem 1.1 is given in Section 2; the key tools are a recent result of Asmussen and Schmidt (1995) on ladder height distributions and a well-known 
association inequality. In that section we also give a counterexample showing that $\psi^{*}<_{\text {so }} \psi$ may fail if (1.7) is violated. In Section 3 we discuss the role of (1.8). Computational results indicate that (1.8) might be not necessary for $\psi^{*}<_{\text {so }} \psi$. From a result of Rolski (1981) it follows that the weaker ordering $\psi^{*}<_{\text {sl }} \psi$ always holds when claim sizes are i.i.d; we give a self-contained proof in Section 4.

The rest of the paper then deals with the adjustment coefficients. We say that a function $\psi \in \mathscr{R}$ admits an exponential tail with adjustment coefficient $\gamma$ if, for $\breve{\mu} \rightarrow \infty$, we have $\psi(u) \sim C \exp (-\gamma u)$ for some $\mathrm{C}>0$. In the subset of functions $\psi^{(1)}, \psi^{(2)} \in \mathscr{R}$ admitting an exponential tail we define $\psi^{(1)}<_{\text {et }} \psi^{(2)}$ iff $\gamma^{(1)}>\gamma^{(2)}$; this ordering criterion is used in a more or less explicit way in much of the actuarial literature (see e.g. Klüppelberg (1993)). Section 5 studies the problem of $\psi^{*}<_{\text {et }} \psi$. We show that this ordering holds at least in the cases when $p=2$ or when (1.6), (1.7) and (1.8) are fulfilled. Section 6 then gives some perturbation analysis for the adjustment coefficient. For example, we compute the first error term in the approximation $\gamma \approx \gamma^{*}$ which is valid when the degree of Markov-modulation is small.

\section{STOCHASTIC ORDERING}

Consider the irreducible Markov process $\{J(t)\}$ described in the preceding section. Let $\left\{J_{i}(t)\right\}$ be the Markov process with the same intensity matrix $\Lambda$ as $\{J(t)\}$, but starting in state $i$, i.e. $J_{i}(0)=i$, and $\left\{N_{i}(t)\right\}$ be the counting process with intensity process $\left\{\beta_{\mathrm{J}_{i}(\mathrm{t})}\right\}$. As before, define $\left\{S_{i}(t)\right\}_{t \geq 0}=\left\{\sum_{k=1}^{N_{i}(t)} U_{i, k}-t\right\}_{t \geq 0}$ as the associated surplus process with arrival process $\left\{N_{i}(t)\right\}$ and claim sizes $U_{i, k}$, respectively, and with the ruin function $\psi_{i}(u)=\mathbb{P}\left(\sup _{t \geq 0} S_{i}(t)>u\right)$.

Lemma 2.1 Assume that conditions (1.6), (1.7) and (1.8) are fulfilled. Then, for $i<j$, it holds that $\psi_{i}<_{\text {so }} \psi_{j}$.

Proof. Let $i<j$. Because of (1.8), using Theorem 4.2.8 and Proposition 1.10.4 of Stoyan (1983), we can assume that $J_{i}(t) \leq J_{j}(t)$ for all $t \geq 0$, which implies that the intensities of $N_{i}$ and $N_{j}$ are pathwise ordered. Therefore, we can assume that any jump epoch, say the $k$ th occurring at time $t_{k}$, for $N_{i}$ is also a jump epoch for $N_{j}$, say the $l$ th, where we use the notation $l=l_{\mathrm{k}}$. Since $J_{i}\left(t_{k}\right) \leq J_{j}\left(t_{k}\right)$, it follows from (1.7) that the claim sizes can be chosen in such a way that $U_{i, k} \leq U_{j, l_{k}}$ with probability one and hence

$$
S_{j}(t) \geq \sum_{k: t_{k} \leq t} U_{j, l_{k}}-t \geq \sum_{k: t_{k} \leq t} U_{i, k}-t=S_{i}(t)
$$

From this the ordering $\psi_{i}<_{\text {so }} \psi_{j}$ easily follows.

Let $\tau_{+}$be the first ladder epoch of the surplus process $\{S(t)\}$, i.e. $\tau_{+}=\inf \{t>0$ : $S(t)>0\}$. Furthermore, we consider the ladder height $Z_{+}=\lim _{t} \downarrow_{+} S_{t}$ provided that $\tau_{+}<\infty$. Specializing Corollary 1 of Asmussen and Schmidt (1995), we have the following result. 
Lemma 2.2 For the Markov-modulated surplus process $\{S(t)\}$,

(a) $\mathbb{P}\left(\tau_{+}<\infty\right)=\rho=\sum \pi_{i} \beta_{i} \mu_{i}$;

(b) the conditional distribution of $J\left(\tau_{+}\right)$given $\tau_{+}<\infty$ is obtained by the probabilities

$$
\pi_{i}^{(L)}=\frac{\pi_{i}^{(P)} \mu_{i}}{\sum \pi_{j}^{(P)} \mu_{j}}
$$

where $\left\{\pi_{i}^{P}\right\}$ with $\pi_{i}^{(P)}=\frac{\pi_{i} \beta_{i}}{\sum \pi_{j} \beta_{j}}$ is the (Palm) initial distribution of $\{J(t)\}$ (at a typical jump epoch of $\{N(t)\})$;

(c) the conditional distribution of the ladder height $Z_{+}$given $\tau_{+}<\infty, J\left(\tau_{+}\right)=i$ has the density $\bar{B}_{i}(x) / \mu_{i}$;

(d) the conditional distribution $G$ of the ladder height $Z_{+}$given $\tau_{+}<\infty$, has the density $\bar{B}^{*}(x) / \mu$.

Note in particular that the ruin probability $\mathbb{P}\left(\tau_{+}<\infty\right)$ and the ladder height distribution are the same for the Markov-modulated model and the averaged compound Poisson one.

In the proof of Theorem 1.1 we will use, besides Lemma 2.1 and Lemma 2.2, the following standard result going back to Chebyshev and appearing, for instance, in Mitrinović et al. (1993), see also Esary et al. (1967):

Lemma 2.3 If $a_{1} \leq \ldots \leq a_{p}, b_{1} \leq \ldots \leq b_{p}$ and $\pi_{i}>0(i=1, \ldots, p), \sum_{i=1}^{\mathrm{p}} \pi_{\mathrm{i}}=1$ then

$$
\sum_{i=1}^{p} \pi_{i} a_{i} b_{i} \geq \sum_{i=1}^{p} \pi_{i} a_{i} \sum_{j=1}^{p} \pi_{j} b_{j}
$$

where the equality holds if and only if $a_{1}=\ldots=a_{p}$ or $b_{1}=\ldots=b_{p}$.

Proof of Theorem 1.1 Conditioning upon the first ladder epoch, from Lemma 2.2 we obtain

$$
\begin{aligned}
\psi^{*}(u) & =\rho \bar{G}(u)+\beta^{*} \int_{0}^{u} \psi^{*}(u-x) \bar{B}^{*}(x) d x \\
\psi(u) & =\rho \bar{G}(u)+\rho \sum_{i=1}^{p} \int_{0}^{u} \psi_{i}(u-x) \pi_{i}^{(L)} \bar{B}_{i}(x) / \mu_{i} d x \\
& =\rho \bar{G}(u)+\int_{0}^{u} \sum_{i=1}^{p} \pi_{i} \beta_{i} \bar{B}_{i}(x) \psi_{i}(u-x) d x \\
& \geq \rho \bar{G}(u)+\int_{0}^{u} \sum_{i=1}^{p} \pi_{i} \beta_{i} \bar{B}_{i}(x) \cdot \sum_{i=1}^{p} \pi_{i} \psi_{i}(u-x) d x \\
& =\rho \bar{G}(u)+\beta^{*} \int_{0}^{u} \psi(u-x) \bar{B}^{*}(x) d x,
\end{aligned}
$$

where the inequality in (2.2) follows by considering the increasing functions $\beta_{i} \vec{B}_{i}(x)$ and $\psi_{i}(u-x)$ of $i$ and using Lemma 2.3. Comparing (2.2) and (2.1), it follows by a standard argument from renewal theory that $\psi$ dominates the solution $\psi^{*}$ of the renewal equation $(2.1)$. 
The following result shows that $\psi^{*}<_{\text {so }} \psi$ may fail if $(1.7)$ is violated.

Proposition 2.1 Assume that $\beta_{i} \mu_{i}<1$ for all $i$, that

$$
\sum_{i=1}^{p} \pi_{i} \beta_{i}^{2} \mu_{i}<\sum_{i=1}^{p} \pi_{i} \beta_{i} \cdot \sum_{i=1}^{p} \pi_{i} \beta_{i} \mu_{i}
$$

and that $\boldsymbol{\Lambda}$ has the form $\varepsilon \Lambda_{0}$ for some fixed intensity matrix $\Lambda_{0}$. Then $\psi^{*}<_{\text {so }} \psi$ fails for all sufficiently small $\varepsilon>0$.

Proof. From the defining equations (1.1) it follows that the ruin functions $\psi(u)$ and $\psi^{*}(u)$ are right continuous at $u=0$. Since the tail functions $\bar{B}^{*}$ and $\bar{B}_{i}$ also possess this property, from (2.1) and (2.2) we get that the right derivatives $\frac{d^{+} \psi^{*}}{d u}(0)$ and $\frac{d^{+} \psi}{d u}(0)$ exist and are given by

$$
\begin{aligned}
& \frac{d^{+} \psi^{*}}{d u}(0)=-\rho / \mu^{*}+\beta^{*} \psi^{*}(0)=\sum_{i=1}^{p} \pi_{i} \beta_{i} \cdot \sum_{i=1}^{p} \pi_{i} \beta_{i} \mu_{i}-\beta^{*} \\
& \frac{d^{+} \psi}{d u}(0)=\sum_{i=1}^{p} \pi_{i} \beta_{i} \psi_{i}(0)-\beta^{*}
\end{aligned}
$$

Since $\psi(0)=\psi^{*}(0)$, it is sufficient to show that $\frac{d^{+} \psi}{d u}(0)<\frac{d^{+} \psi^{*}}{d u}(0)$ for $\varepsilon$ small enough. From a well-known continuity property of stationary single-server queues (see e.g. Theorem 3.2.1 of Franken et al. (1982)) it follows that $\psi_{i}(u)$ converges to the corresponding ruin probability for the compound Poisson model with parameters $\beta_{i}, B_{i}$ as $\varepsilon \downarrow 0$. For $u=0$, this limiting ruin probability is $\beta_{i} \mu_{i}$. Consequently, by (2.3) and (2.4) we get that

$$
\lim _{\varepsilon \downarrow 0}\left(\frac{d^{+} \psi^{*}}{d u}(0)-\frac{d^{+} \psi}{d u}(0)\right)=\sum_{i=1}^{p} \pi_{i} \beta_{i} \sum_{i=1}^{p} \pi_{i} \beta_{i} \mu_{i}-\sum_{i=1}^{p} \pi_{i} \beta_{i} \lim _{\varepsilon \downarrow 0} \psi_{i}(0)>0 .
$$

This finishes the proof.

To see that the condition of Proposition 2.1 is not vacuous, we briefly mention an example for which (2.3) is fulfilled. Let

$$
p=2, \pi=(1 / 2,1 / 2), \beta_{1}=10^{-3}, \beta_{2}=1, \mu_{1}=10^{2}, \mu_{2}=10^{-4} \text {. }
$$

Then the left side of (2.3) is of order $10^{-4}$, whereas the right side of (2.3) is of order $10^{-1}$.

We finally remark that, besides Theorem 1.1 , a further related result holds. Consider the sequence of consecutive ladder epochs $\tau_{+}^{(1)}, \tau_{+}^{(2)}, \ldots$ of the surplus process $\{S(t)\}$. Note that $\tau_{+}^{(1)}=\tau_{+}$.

Proposition 2.2 Assume that conditions (1.6), (1.7) and (1.8) hold. Then, for every $k=1,2, \ldots$,

$$
\rho^{k} \leq \mathbb{P}\left(\tau_{+}^{(1)}<\infty, \ldots, \tau_{+}^{(k)}<\infty\right)
$$


Proof. Proceeding analogously as in the proof of Lemma 2.1 we get that, for each $i=1, \ldots, p$,

$$
\mathbb{P}\left(\tau_{+}^{(1)}<\infty, \ldots, \tau_{+}^{(k)}<\infty\right) \leq \mathbb{P}\left(\tau_{+}^{(1)}<\infty, \ldots, \tau_{+}^{(k)}<\infty \mid J(0) \geq i\right)
$$

and, equivalently,

$$
\mathbb{P}(J(0) \geq i) \leq \mathbb{P}\left(J(0) \geq i \mid \tau_{+}^{(1)}<\infty, \ldots, \tau_{+}^{(k)}<\infty\right) .
$$

On the other hand, from Lemma 2.2 we obtain

$$
\begin{aligned}
& \mathbb{P}\left(\tau_{+}^{(1)}<\infty, \ldots, \tau_{+}^{(k)}<\infty\right) \\
& =\sum_{i=1}^{p} \mathbb{P}\left(\tau_{+}^{(1)}<\infty, J\left(\tau_{+}^{(1)}\right)=i\right) \mathbb{P}\left(\tau_{+}^{(2)}<\infty, \ldots, \tau_{+}^{(k)}<\infty \mid \tau_{+}^{(1)}<\infty, J\left(\tau_{+}^{(1)}\right)=i\right) \\
& =\sum_{i=1}^{p} \pi_{i} \beta_{i} \mu_{i} \mathbb{P}\left(\tau_{+}^{(1)}<\infty, \ldots, \tau_{+}^{(k-1)}<\infty \mid J(0)=i\right) \\
& =\mathbb{P}\left(\tau_{+}^{(1)}<\infty, \ldots, \tau_{+}^{(k-1)}<\infty\right) \sum_{i=1}^{p} \beta_{i} \mu_{i} \mathbb{P}\left(J(0)=i \mid \tau_{+}^{(1)}<\infty, \ldots, \tau_{+}^{(k-1)}<\infty\right) .
\end{aligned}
$$

Observe that $\beta_{1} \mu_{1} \leq \beta_{2} \mu_{2} \leq \ldots \leq \beta_{p} \mu_{p}$. Thus, from (2.6) and from Theorem 1.2.2 of Stoyan (1983), we have

$$
\rho=\sum_{i=1}^{p} \beta_{i} \mu_{i} \mathbb{P}(J(0)=i) \leq \sum_{i=1}^{p} \beta_{i} \mu_{i} \mathbb{P}\left(J(0)=i \mid \tau_{+}^{(1)}<\infty, \ldots, \tau_{+}^{(k-1)}<\infty\right) .
$$

Consequently,

$$
\mathbb{P}\left(\tau_{+}^{(1)}<\infty, \ldots, \tau_{+}^{(k)}<\infty\right) \geq \rho \mathbb{P}\left(\tau_{+}^{(1)}<\infty, \ldots, \tau_{+}^{(k-1)}<\infty\right)
$$

and (2.5) follows by induction.

Note that, by using a corresponding result of queueing theory, still another type of conditions can be given for the validity of (1.2). These conditions are formulated in terms of aging properties of the interarrival time distribution. Such aging properties were introduced in reliability theory, see e.g. Barlow and Proschan (1975). If interarrival times are i.i.d., if their distribution function $F$ with mean $\left(\beta^{*}\right)^{-1}$ has the property NWUE (that is $\int_{t}^{\infty} \bar{F}(x) d x \geq\left(\beta^{*}\right)^{-1} \bar{F}(t)$ for all $t \geq 0$ ) and if claim sizes are also i.i.d. and independent of arrivals, then $\psi^{*}<_{\text {so }} \psi$ holds with $\psi^{*}$ defined by the Poisson compound model with arrival rate $\beta^{*}$ and the same claim sizes, see e.g. Remark 4.6.13 in Franken et al. (1982). In this way, the validity of the ordering $\psi^{*}<_{\text {so }} \psi$ can be verified for a further model with special doubly stochastic Poisson arrival process and i.i.d. claim sizes. This is when the stochastic arrival-intensity process is assuming only two values: zero and one positive. It is well-known that, in this model, interarrival times are i.i.d. provided that the consecutive sojourn times of the environment process in its two states form an alternating renewal process and that the sojourn times in the state with positive arrival intensity are exponentially distributed. Furthermore, it is easy to see that then the distribution of interarrival times has the property NWUE. Thus, from the remark above, $\psi^{*}<_{\text {so }} \psi$ follows. In Rolski (1981) this was noticed under the additional assumption that the distribution of sojourn times in the zero state has the stronger aging property DFR. 


\section{EXPONENTIAL CLAIM SIZES}

Now we discuss the role of the monotonicity condition (1.8) considering the special case (1.9), i.e. the distribution function $B$ of claim sizes does not depend on the actual state of the environmental process $\{J(t)\}$. Moreover, we assume that the claim sizes are exponentially distributed with expectation one, i.e.

$$
B(x)=1-\mathrm{e}^{-x} \quad \text { for every } x \geq 0 .
$$

Note that, in this case, a different proof of Theorem 1.1 can be given by using Proposition 2.2. This follows from the fact that, for claim sizes with the standard exponential distribution, the ruin function $\psi(u)$ can be represented in the form

$$
\begin{aligned}
\psi(u) & =\sum_{k=1}^{\infty}\left(f_{u}(k)-f_{u}(k-1)\right) \mathbb{P}\left(\tau_{+}^{(1)}<\infty, \ldots, \tau_{+}^{(k)}<\infty\right) \\
& =e^{-u} \sum_{k=1}^{\infty} \frac{u^{k}}{k !} \mathbb{P}\left(\tau_{+}^{(1)}<\infty, \ldots, \tau_{+}^{(k)}<\infty\right)
\end{aligned}
$$

where $f_{u}(k)=\mathbb{P}\left(\sum_{i=1}^{k} M_{i}>u\right)$ for a fixed $u \geq 0$, and $\mathbf{M}_{1}, \mathrm{M}_{2}, \ldots$ are i.i.d. random variables whose distribution function is given by (3.1). Clearly, $f_{u}(k)-f_{u}(k-1) \geq 0$. Thus, assuming that (1.6), (1.8), (1.9) and (3.1) hold, from Proposition 2.2 we obtain

$$
\psi(u) \geq \sum_{k=1}^{\infty}\left(f_{u}(k)-f_{u}(k-1)\right) \rho^{k}=\psi^{*}(u) .
$$

Moreover, because the ruin probability $\psi(u)$ is equal to $\mathbb{P}(V>u)$, where $V$ is the stationary virtual waiting time in a single-server queue with the same, but timereversed input (see e.g. Asmussen (1989)), from Theorem 6.2.1 of Neuts (1981) we get that, under (1.9) and (3.1),

$$
\psi(u)=\sum_{k=1}^{\infty}\left(f_{u}(k)-f_{u}(k-1)\right) \pi \boldsymbol{R}^{k} \boldsymbol{e},
$$

where $\boldsymbol{R}$ is the minimal nonnegative solution of the matrix equation

$$
R^{2}+R(C-I-\Delta)+\Delta=\mathbf{0},
$$

$\Delta$ is the $p \times p$ diagonal matrix with diagonal elements $\beta_{1}, \ldots, \beta_{\mathrm{p}}, \boldsymbol{I}=\left(\boldsymbol{\delta}_{i j}\right)$ the $p \times p$ identity matrix, $e$ the p-dimensional column vector of ones, and $C$ the intensity matrix of the stationary Markov process $\{\tilde{J}(t)\}$ obtained, after reversion of time, from the stationary Markov process $\{J(t)\}$ governed by $\Lambda$, i.e. $\tilde{J}(t)=J(-t)$. From (3.2) and (3.4) we have

$$
\pi \boldsymbol{R}^{k} \boldsymbol{e}=\mathbb{P}\left(\tau_{+}^{(1)}<\infty, \ldots, \tau_{+}^{(k)}<\infty\right)
$$

In the following we want to discuss the question whether the monotonicity condition (1.8) is necessary for $\psi^{*} \prec_{\text {so }} \psi$. One of the possible approaches to verify this is to parameterize $\Delta$ in the following way. Let $\Delta(x)$ be the $p \times p$ diagonal 
matrix with the $i$ th diagonal element, $\beta_{i}(x)=\beta^{*}+c_{i} x$ where the constants $c_{i}$ are such that $\sum_{i=1}^{p} \pi_{i} c_{i}=0$. Furthermore, let $\boldsymbol{R}(x)$ be the corresponding minimal solution of (3.5). Clearly $x=0$ yields the compound Poisson case. The goal is to prove the inequality

$$
\boldsymbol{\pi} \boldsymbol{R}^{k} \boldsymbol{e} \geq \rho^{k} \text { for all } k \geq 0
$$

without using assumption (1.8), i.e. to show that

$$
\min _{\left\{x: \beta_{i}(x) \geq 0 \forall i\right\}} \pi \boldsymbol{R}^{k}(x) \boldsymbol{e}=\boldsymbol{\pi} \boldsymbol{R}^{k}(0) \boldsymbol{e}=\left(\boldsymbol{\beta}^{*}\right)^{k} \quad \text { for all } k \geq 0 .
$$

Computations as in the following example even leads to the conjecture that $\pi \boldsymbol{R}^{k}(x) \boldsymbol{e}$ is a convex function of $x$ taking its minimum at $x=0$.

Example 3.1 Let $\beta^{*}=0.5$,

$$
\boldsymbol{C}=\left(\begin{array}{ccc}
-20 & 0 & 20 \\
20 & -40 & 20 \\
30 & 30 & -60
\end{array}\right) \quad \text { and } \quad \Delta(x)=\left(\begin{array}{ccc}
\beta^{*}+x & 0 & 0 \\
0 & \beta^{*}-0.5 x & 0 \\
0 & 0 & \beta^{*}-1.875 x
\end{array}\right)
$$

Then, $\pi=\left(\frac{9}{16}, \frac{3}{16}, \frac{4}{16}\right)$ and the intensity matrix $\Lambda$ of the corresponding timereversed Markov process is given by (see e.g. Theorem 1.12 of Kelly (1979))

$$
\boldsymbol{\Lambda}=\left(\begin{array}{ccc}
-20 & \frac{20}{3} & \frac{40}{3} \\
0 & -40 & 40 \\
45 & 15 & -60
\end{array}\right)
$$

which does not satisfy (1.8). However, from numerical computations one gets the following picture for $k=2$ (see Figure 3.I).

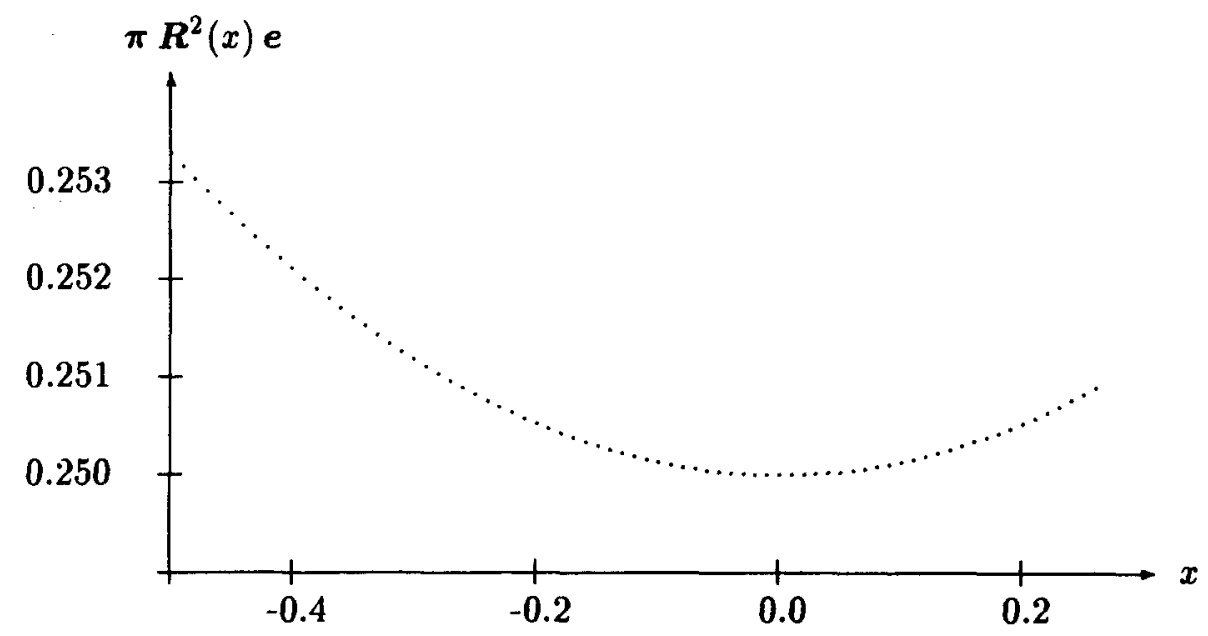

FIGURE 3.1. 
One possibility to prove (3.7) is to investigate the first two derivatives $g_{k}^{\prime}(x)$ and $g_{k}^{\prime \prime}(x)$ of $g_{k}(x)=\pi R^{\mathrm{k}}(x) e$ with respect to $x$. A first step into this direction is the following result. However, it seems to be impossible to evaluate the second derivative of $g_{k}(x)$ explicitly.

Proposition 3.1 For every $k=1,2, \ldots$, we have

$$
\begin{gathered}
g_{k}^{\prime}(0)=0 \\
g_{k}^{\prime \prime}(0)=2\left(\beta^{*}\right)^{k-1} \sum_{i=1}^{k-1} \pi\left(\frac{\boldsymbol{R}^{i}}{\beta^{*}}\right)^{\prime}(0) \boldsymbol{\Delta}^{\prime}(0) \boldsymbol{e} .
\end{gathered}
$$

Proof. The assertion follows by induction with respect to $k$ using the facts that

$$
\pi \boldsymbol{R}(0)=\beta^{*} \pi, \Delta(0) e=\beta^{*} e, \Delta^{\prime \prime}(0) e=0
$$

$$
\boldsymbol{R}(x) \boldsymbol{e}=\boldsymbol{\Delta}(x) \boldsymbol{e}
$$

hold (see Section 6.2 of Neuts (1981)). In particular, from (3.11) and (3.10) we get

$$
\begin{aligned}
g_{k}^{\prime}(0) & =\left.\left(\pi \boldsymbol{R}^{k-1}(x) \boldsymbol{\Delta}(x) \boldsymbol{e}\right)^{\prime}\right|_{x=0} \\
& =\boldsymbol{\pi}\left(\boldsymbol{R}^{k-1}\right)^{\prime}(0) \boldsymbol{\beta}^{*} \boldsymbol{e}+\left(\boldsymbol{\beta}^{*}\right)^{k-1} \boldsymbol{\pi} \boldsymbol{\Delta}^{\prime}(0) \boldsymbol{e} \\
& =\boldsymbol{\beta}^{*} g_{k-1}^{\prime}(0),
\end{aligned}
$$

because $\pi \boldsymbol{\Lambda}^{\prime}(0)=\sum_{i=1}^{p} \pi_{i} c_{i}=0$. Thus, by induction,

$$
g_{k}^{\prime}(0)=\left(\beta^{*}\right)^{k-1} g_{1}^{\prime}(0)=\left(\beta^{*}\right)^{k-1} \pi \Delta^{\prime}(0)=0 .
$$

In order to prove (3.9) we can proceed similarly. Namely,

$$
\begin{aligned}
g_{k}^{\prime \prime}(0) & =\left(\pi \boldsymbol{R}^{k-1}(x) \boldsymbol{\Delta}(x) \boldsymbol{e}\right)^{\prime \prime} \\
& =\pi\left(\boldsymbol{R}^{k-1}\right)^{\prime \prime}(0) \beta^{*} \boldsymbol{e}+2 \pi\left(\boldsymbol{R}^{k-1}\right)^{\prime}(0) \boldsymbol{\Delta}^{\prime}(0) \boldsymbol{e}+\boldsymbol{\pi} \boldsymbol{R}^{k-1}(0) \boldsymbol{\Delta}^{\prime \prime}(0) \boldsymbol{e} \\
& =\beta^{*} g_{k-1}^{\prime \prime}(0)+2 \pi\left(\boldsymbol{R}^{k-1}\right)^{\prime}(0) \boldsymbol{\Delta}^{\prime}(0) \boldsymbol{e} \\
& \vdots \\
& =2 \pi\left(\sum_{i=1}^{k-1}\left(\beta^{*}\right)^{i-1}\left(\boldsymbol{R}^{k-i}\right)^{\prime}(0)\right) \boldsymbol{\Delta}^{\prime}(0) \boldsymbol{e} .
\end{aligned}
$$

Another possible way to verify whether the monotonicity condition (1.8) is necessary could be to use a result from queueing theory, i.e. to utilize Theorem 4.1 from Chang and Nelson (1993), in particular their formula (32), where they considered a single-server queue with a doubly stochastic Poisson arrival process $\{N(t)\}$ with intensity process $\left\{\beta_{\varepsilon \tilde{J}(t)}\right\}$ and showed that for large $\varepsilon$ the second-order approximation for the expected stationary queue length is given by

$$
\mathbb{E} L(0)=\frac{\beta^{*}}{1-\beta^{*}}+\varepsilon^{-1} \frac{1}{1-\beta^{*}} \pi S \Delta \boldsymbol{e}+\mathrm{O}\left(\varepsilon^{-2}\right),
$$

where $S$ is a certain $p \times p$ matrix which is determined by $C$ and $\Delta$ (or, equivalently, by $\boldsymbol{\Lambda}$ and $\boldsymbol{\Delta}$ ). The question is whether it is possible to find an example such that $\pi S \Delta e<0$. In that case, $\mathbb{E} L(0)<\frac{\beta^{*}}{1-\beta^{*}}$ for sufficiently large $\varepsilon$ and hence (3.6) 
would not be true. Moreover, from Little's and Takacs' formulas (see (4.4)), it would follow that then $\int_{0}^{\infty} \psi(u) d u<\int_{0}^{\infty} \psi^{*}(u) d u$. Thus, also $\psi^{*}<_{\text {so }} \psi$ would not be true in general. Chang and Nelson (1993) proved that $\pi S \Delta e \geq 0$ provided that $\{\tilde{J}(t)\}$ is dynamically reversible, where a stationary Markov process with intensity matrix $\boldsymbol{\Lambda}$ is dynamically reversible if there exists a permutation of $\{1,2, \ldots, m\}$, denoted by $j \rightarrow j^{+}, \mathrm{j}=1,2, \ldots, m$, such that (i) $\pi_{j}=\pi_{j}+$, (ii) $\Lambda_{j j}=\Lambda_{j}+^{+}$and (iii) $\pi_{i} \Lambda_{i j}=\pi+\Lambda_{j}+_{j}+$. Therefore, we tried to find $\underset{\sim}{\sim}$ example such that $\pi S \Delta e<0$ for neither $\{J(t)\}$ stochastically monotone nor $\{\tilde{J}(t)\}$ dynamically reversible but we could not get it in none of the 20 million different configurations which we calculated numerically. Moreover, our computations (see also Example 3.2) yield the conjecture that the expected queue length $\mathbb{E} L(0)$ is always strictly decreasing in $\varepsilon$, at least for sufficiently large $\varepsilon$ where no additional assumptions are needed (like dynamical reversibility of $\{\tilde{J}(t)\}$ or stochastic monotonicity of $\{J(t)\})$.

Example 3.2 Let

$$
\boldsymbol{C}=\left(\begin{array}{ccc}
-2 & 0 & 2 \\
2 & -4 & 2 \\
3 & 3 & -6
\end{array}\right), \quad \boldsymbol{\Delta}=\left(\begin{array}{ccc}
0.3 & 0 & 0 \\
0 & 0.6 & 0 \\
0 & 0 & 0.875
\end{array}\right)
$$

Note that the Markov process $\{\tilde{J}(t)\}$ with this intensity matrix $C$ is not dynamically reversible, because there is no permutation which fulfills conditions (i)-(iii) above. Also, the corresponding time-reversed process $\{J(t)\}$ is not stochastically monotone (see Example 3.1). But, numerical computations show (see Figure 3.2 ) that $\boldsymbol{\pi} \boldsymbol{R}^{2}(\varepsilon) \boldsymbol{e}$ is monotonously decreasing in $\varepsilon$, where $\boldsymbol{R}(\varepsilon)$ is the minimal solution of $\boldsymbol{R}^{2}(\varepsilon)+\boldsymbol{R}(\varepsilon)(\varepsilon \boldsymbol{C}-\boldsymbol{I}-\boldsymbol{\Delta})+\Delta=\mathbf{0}$.

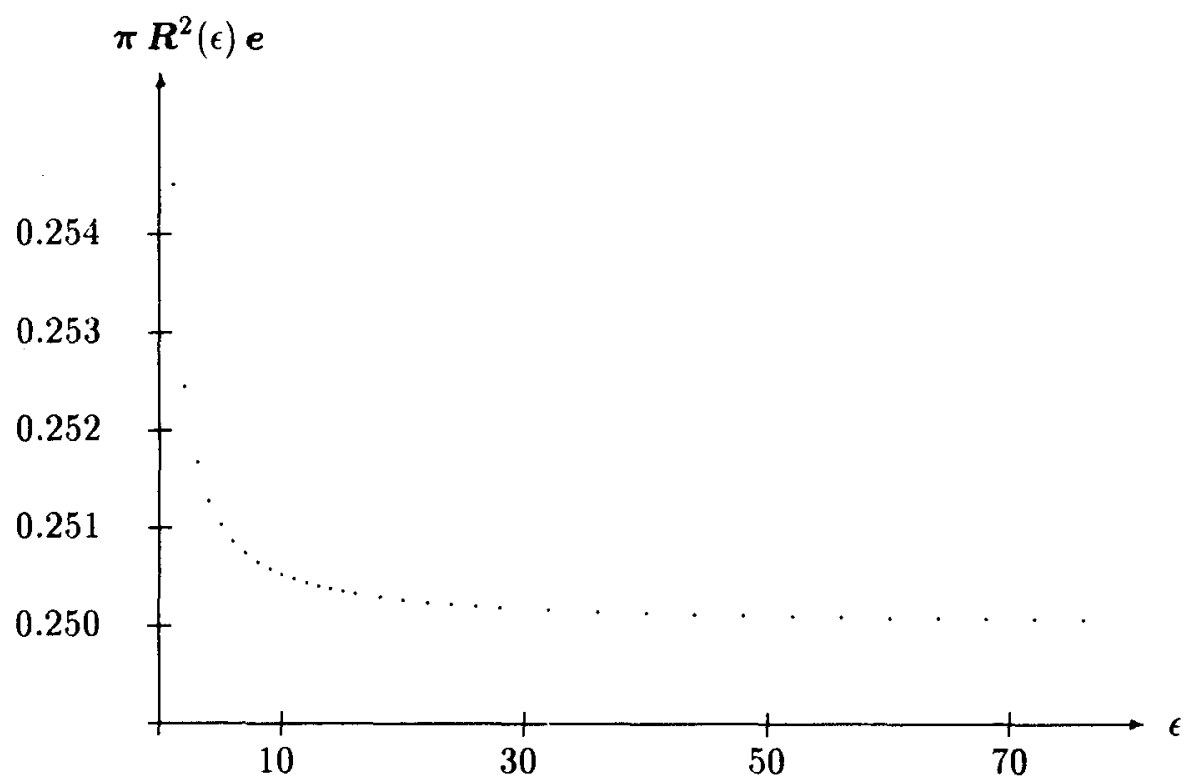

FIGURE 3.2. 


\section{STOP-LOSS ORDERING}

In this section we consider the case that claims arrive according to a doubly stochastic Poisson process $\{N(t)\}$ with an arbitrary stationary ergodic intensity process $\{\beta(t)\}$ such that $\beta^{*}=\mathbb{E} \beta(0)<\infty$. In particular, we do not assume that $\{\beta(t)\}$ is generated by a Markov process as it was assumed in the preceding sections. By some authors, such a more general counting process $\{N(t)\}$ is called a Cox process. Let the sequence $\left\{U_{k}\right\}$ of consecutive claim sizes consist of i.i.d. random variables with distribution function $B$ and assume that they are also independent of arrivals and that $\beta^{*} \mathbb{E} U_{k}<1$. Under these assumptions an interesting comparability property was derived in Rolski (1981) concerning convex ordering of virtual waiting times in single-server queues. We restate this result here, but now in the risk theoretical setting, and we give a self-contained proof. Like in Sections 1 to 3 , by $\psi(u)$ we denote the ruin probability defined in (1.1) and (1.4) and by $\psi^{*}(u)$ the corresponding ruin probability for the associated averaged compound Poisson model, that is with Poisson arrivals with rate $\beta^{*}$ and with the same claim sizes $U_{k}$.

Theorem 4.1 Let the claim arrival process $\{N(t)\}$ be an arbitrary Cox process with stationary ergodic intensity process $\{\beta(t)\}$, let the claim sizes $U_{k}$ be i.i.d. and independent of $\{N(t)\}$, and $\mathbb{E} \beta(t) \mathbb{E} U_{k}<1$. Then,

$$
\psi^{*}<_{\mathrm{sl}} \psi
$$

In the proof of Theorem 4.1 we use the following lemma, which seems to be also of independent interest and where the ruin function $\psi(u)$ appearing in (4.1) is replaced by a Palm-type analogue. By $\psi^{\circ}(u)$ we denote the ruin function given by

$$
\psi^{\circ}(u)=\mathbb{P}\left(\sup _{t \geq 0} S^{\circ}(t)>u\right), \quad S^{\circ}(t)=\sum_{k=1}^{N^{\circ}(t)} U_{k}-t,
$$

where $\left\{N^{\circ}(t)\right\}$ is the (reduced) Palm version of the stationary Cox process $\{N(t)\}$. It is well-known that $\left\{N^{\circ}(t)\right\}$ again is a Cox process. Its intensity process we denote by $\left\{\beta^{\circ}(t)\right\}$. Moreover, it holds

$$
\mathbb{E} \Gamma(t)=\frac{t}{\beta^{*}} \text { for every } t \geq 0,
$$

where $\Gamma(t)=\inf \left\{u \geq 0: \int_{0}^{u} \beta^{\circ}(s) d s \geq t\right\}$, see Chapter 5 of König and Schmidt (1992).

Lemma 4.1 Under the assumptions of Theorem 4.1, the ruin functions $\psi^{\circ}(u)$ and $\psi^{*}(u)$ are related by

$$
\psi^{*}<_{\mathrm{sl}} \psi^{\circ}
$$

Proof. It is easy to see that the jump epochs of $\left\{N^{\circ}(t)\right\}$ can be represented by the sequence $\Gamma\left(M_{1}\right), \Gamma\left(M_{1}+M_{2}\right), \ldots$ where $M_{1}, M_{2}, \ldots$ are i.i.d. random variables which are independent of $\left\{\beta^{\circ}(t)\right\}$ and whose distribution function is given by (3.1). Thus, from Jensen's inequality for conditional expectations and from (4.2) we get 


$$
\begin{aligned}
& \int_{x}^{\infty} \psi^{\circ}(u) d u=\int_{x}^{\infty} \mathbb{P}\left(\sup _{t \geq 0} S^{\circ}(t)>u\right) d u \\
& \left.=\int_{x}^{\infty} \mathbb{P}\left(\max 0, U_{1}-\Gamma\left(M_{1}\right), U_{1}+U_{2}-\Gamma\left(M_{1}+M_{2}\right), \ldots\right)>u\right) d u \\
& =\mathbb{E}\left[\max \left(0, U_{1}-\Gamma\left(M_{1}\right), U_{1}+U_{2}-\Gamma\left(M_{1}+M_{2}\right), \ldots\right)-x\right]_{+} \\
& =\mathbb{E}\left[\mathbb{E}\left(\left[\max \left(0, U_{1}-\Gamma\left(M_{1}\right), U_{1}+U_{2}-\Gamma\left(M_{1}+M_{2}\right), \ldots\right)-x\right]_{+} \mid\left\{U_{k}, M_{k}\right\}\right)\right] \\
& \geq \mathbb{E}\left[\max \left(0, U_{1}-\mathbb{E}\left[\Gamma\left(M_{1}\right) \mid\left\{M_{k}\right\}\right], U_{1}+U_{2}-\mathbb{E}\left[\Gamma\left(M_{1}+M_{2}\right) \mid\left\{M_{k}\right\}\right], \ldots\right)-x\right]_{+} \\
& =\mathbb{E}\left[\max \left(0, U_{1}-\frac{M_{1}}{\beta^{*}}, U_{1}+U_{2}-\frac{M_{1}+M_{2}}{\beta^{*}}, \ldots\right)-x\right]_{+} \\
& =\int_{x}^{\infty} \psi^{*}(u) d u,
\end{aligned}
$$

where $[x]_{+}=\max (0, x)$.

Proof of Theorem 4.1. Observe that the ruin functions $\psi(u)$ and $\psi^{\circ}(u)$ satisfy the relationship

$$
\psi(u)=\beta^{*} \int_{0}^{\infty} \psi^{\circ}(u-v)(1-B(v)) d v
$$

which is known as Takacs' formula (see Corollary 4.5.4 of Franken et al. (1982)). Thus,

$$
\begin{aligned}
& \int_{x}^{\infty} \psi(u) d u=\beta^{*} \int_{x}^{\infty} \int_{0}^{\infty} \psi^{\circ}(u-v)(1-B(v)) d v d u \\
& =\beta^{*} \int_{0}^{\infty} \int_{x}^{\infty} \psi^{\circ}(u-v) d u(1-B(v)) d v \\
& \geq \beta^{*} \int_{0}^{\infty} \int_{x}^{\infty} \psi^{*}(u-v) d u(1-B(v)) d v \\
& =\int_{x}^{\infty} \beta^{*} \int_{0}^{\infty} \psi^{*}(u-v)(1-B(v)) d v d u \\
& =\int_{x}^{\infty} \psi^{*}(u) d u,
\end{aligned}
$$

where Lemma 4.1 and the well-known fact has been used that $\psi^{*}(u)$ satisfies the integral equation $\psi^{*}(u)=\beta^{*} \int_{0}^{\infty} \psi^{*}(u-v)(1-B(v)) d v$.

\section{ORDERING OF ADJUSTMENT COEFFICIENTS}

We now consider the subset of ruin functions admitting an exponential tail, i.e. ruin functions $\psi(u)$ such that there exists a $\gamma \in(0, \infty)$ with $0<\lim _{u \rightarrow \infty} \psi(u) e^{\gamma u}<\infty$. The constant $\gamma$ is called the adjustment coefficient of $\psi(u)$. It turns out that, for such functions, stop-loss ordering implies ordering of their adjustment coefficients.

Proposition 5.1 Assume that the functions $\psi^{(1)}, \psi^{(2)} \in \mathscr{R}$ admit exponential tails with adjustment coefficients $\gamma^{(1)}, \gamma^{(2)}>0$, respectively. Then, $\psi^{(1)}<_{\mathrm{s} 1} \psi^{(2)}$ implies $\psi^{(1)}<_{\mathrm{et}} \psi^{(2)}$.

Proof. Clearly, $\psi^{(i)}(u)=C^{(i)} \exp \left(-\gamma^{(i)} u\right)+r^{(i)}(u)$ where $r^{(i)}(u)=o\left(e^{-\gamma^{(i)} u}\right)$ for $i=1,2$. 
Furthermore, because of $\psi^{(1)}<_{\mathrm{sl}} \psi^{(2)}$, we have for all $x \geq 0$

and hence

$$
\begin{aligned}
0 & \leq \int_{x}^{\infty} \psi^{(2)}(u) d u-\int_{x}^{\infty} \psi^{(1)}(u) d u \\
& =\frac{C^{(2)}}{\gamma^{(2)}} e^{-\gamma^{(2)} x}-\frac{C^{(1)}}{\gamma^{(1)}} e^{-\gamma^{(1)} x}+\int_{x}^{\infty}\left(r^{(2)}(u)-r^{(1)}(u)\right) d u
\end{aligned}
$$

$$
e^{\gamma^{(1)} x} \int_{x}^{\infty}\left(r^{(2)}(u)-r^{(1)}(u)\right) d u \geq \frac{C^{(1)}}{\gamma^{(1)}}-\frac{C^{(2)}}{\gamma^{(2)}} e^{-\left(\gamma^{(2)}-\gamma^{(1)}\right) x}
$$

Observe that from $r^{(i)}(x)=o\left(e^{-\gamma^{(i)} x}\right)$ it follows that $\int_{x}^{\infty} r^{(i)}(u) d u=o\left(e^{-\gamma^{(i)} x}\right)$ Assume for the moment that $\gamma^{(1)}<\gamma^{(2)}$. Then, for $x \rightarrow \infty$, the left side of (5.1) would converge to zero, but the right side to $C^{(1) /} \gamma^{(1)}>0$. By this contradiction $\gamma^{(2)} \leq \gamma^{(1)}$ follows.

Note that, in general, we do not know whether the ruin function induced by a Coxian claim arrival process has an exponential tail. However, in some special cases like periodic environment or Markov modulated environment, this is known (see Asmussen (1989), Asmussen and Rolski (1994)).

Hence we return to the ruin functions $\psi(u)$ and $\psi^{*}(u)$ defined in $(1.1)$ and induced by a Markov-modulated environment and by the associated averaged compound Poisson input, respectively. From Theorem 1.1 and Proposition 5.1 we know that $\gamma \leq \gamma^{*}$ for the adjustment coefficients $\gamma$ and $\gamma^{*}$ of $\psi(u)$ and $\psi^{*}(u)$, and we prove that even the strict inequality is true under the assumption that (1.6), (1.7) and (1.8) (or, for $p=2$, under some other technical assumption) hold. Define

$$
\kappa_{i}(s)=\beta_{i}\left(\hat{B}_{i}(s)-1\right)-s, \quad \kappa^{*}(s)=\beta^{*}\left(\hat{B}^{*}(s)-1\right)-s=\sum_{i=1}^{p} \pi_{i} \kappa_{i}(s),
$$

where $\hat{B}(s)$ denotes the moment generating function of a distribution function $B$. Let $\boldsymbol{K}(s)$ be the $p \times p$ diagonal matrix with the diagonal elements $\kappa_{1}(s), \ldots, \kappa_{p}(s)$ and let, as in Asmussen (1989), $K(s)$ be the dominant eigenvalue of the matrix $\boldsymbol{\Lambda}+\boldsymbol{K}(s)$ given by the Perron-Frobenius theorem, $\boldsymbol{h}(s)$ the corresponding right eigenvector. The solutions of $\kappa_{i}(s)=0, \kappa^{*}(s)=0, \kappa(s)=0$ are denoted by $\gamma_{i}, \gamma^{*}$ and $\gamma$, respectively, where $\gamma^{*}$ and $\gamma$ coincide with adjustment coefficients introduced above. In particular, the adjustment coefficient $\gamma^{*}$ for the associated compound Poisson model fulfills

$$
\gamma^{*}=\sum_{i=1}^{p} \pi_{i} \beta_{i}\left(\hat{B}_{i}\left(\gamma^{*}\right)-1\right)
$$

For $s=\gamma$ we write simply $\boldsymbol{h}=\left(h_{1}, \ldots, h_{p}\right)$. Note that $\boldsymbol{h}$ is a positive vector. Clearly

$$
(\Lambda+K(\gamma)) h=0
$$

and so

It is also clear that

$$
\gamma=\inf \{s>0: \operatorname{det}(\Lambda+K(s))=0\}
$$

$$
\min _{i=1, \ldots, p} \gamma_{i} \leq \gamma \leq \gamma^{*} \leq \max _{i=1, \ldots, p} \gamma_{i}
$$


Proposition 5.2 Assume $p=2$. If $\gamma_{1} \neq \gamma_{2}$ then

$$
\min \left\{\gamma_{1}, \gamma_{2}\right\}<\gamma<\gamma^{*}<\max \left\{\gamma_{1}, \gamma_{2}\right\}
$$

Proof. Let $\lambda_{i}=-\Lambda_{i i}$; then $\pi_{1}=\lambda_{2} /\left(\lambda_{1}+\lambda_{2}\right), \pi_{2}=\lambda_{1} /\left(\lambda_{1}+\lambda_{2}\right)$. Thus

$$
\begin{aligned}
\operatorname{det}(\boldsymbol{\Lambda}+\boldsymbol{K}(s)) & =\operatorname{det}\left(\begin{array}{cc}
-\lambda_{1}+\kappa_{1}(s) & \lambda_{1} \\
\lambda_{2} & -\lambda_{2}+\kappa_{2}(s)
\end{array}\right) \\
& =\kappa_{1}(s) \kappa_{2}(s)-\lambda_{1} \kappa_{2}(s)-\lambda_{2} \kappa_{1}(s) \\
& =\kappa_{1}(s) \kappa_{2}(s)-\left(\lambda_{1}+\lambda_{2}\right) \kappa^{*}(s) .
\end{aligned}
$$

Without loss of generality assume that $\gamma_{1}<\gamma_{2}$. Now distinguish two cases:

Case $0<\gamma_{1}<\gamma_{2}$. Then $\kappa_{i}(s)<0,0<s<\gamma_{i}, \kappa_{i}(s)>0, s>\gamma_{i}$, and hence we get the following table of the sign variation

\begin{tabular}{|c|c|c|c|}
\hline$s \in$ & $\left(0, \gamma_{1}\right)$ & $\left(\gamma_{1}, \gamma^{*}\right)$ & $\left(\gamma^{*}, \gamma_{2}\right)$ \\
\hline$\kappa_{1} \kappa_{2}$ & + & - & - \\
\hline$-\left(\lambda_{1}+\lambda_{2}\right) \kappa^{*}$ & + & + & - \\
\hline
\end{tabular}

which shows that the first zero $\gamma$ of (5.5) is in $\left(\gamma_{1}, \gamma^{*}\right)$.

Case $\gamma_{1}<0<\gamma_{2}$. Then $\kappa_{2}(s)<0,0<s<\gamma_{2}, \kappa_{1}(s)>0, s>\gamma_{1}$, and the sign variation becomes

\begin{tabular}{|c|c|c|}
\hline$s \in$ & $\left(0, \gamma^{*}\right)$ & $\left(\gamma^{*}, \gamma_{2}\right)$ \\
\hline$\kappa_{1} \kappa_{2}$ & - & - \\
\hline$-\left(\lambda_{1}+\lambda_{2}\right) \kappa^{*}$ & + & - \\
\hline
\end{tabular}

so that the first zero is in $\left(0, \gamma^{*}\right)$.

The cases where one of the inequalities is an equality are easily treated in a similar way.

We now turn to the ordering of the adjustment coefficients if $p>2$.

Theorem 5.1 Assume that (1.6), (1.7), (1.8) hold. Then

$$
\gamma^{*}>\gamma
$$

We proceed the proof by a lemma of independent interest.

Lemma 5.1 Under the assumptions of Theorem 5.1, not all $h_{\mathrm{i}}(i=1, \ldots, p)$ are equal.

Proof. It follows from Theorem 6.1 of Asmussen (1989) that there exists a constant $C \in[0, \infty)$ such that

$$
\lim _{u \rightarrow \infty} e^{\gamma u} \psi_{i}(u)=C h_{i}
$$


Thus it is clear by Lemma 2.1 that $h_{1} \leq \ldots \leq h_{p}$. Assume on the contrary that all $h_{i}$ 's are equal. Then $\boldsymbol{\Lambda} \boldsymbol{h}=\mathbf{0}$ and from (5.3) we would have $\beta_{i}\left(\hat{B}_{i}(\gamma)-1\right)=\gamma$ for each $i=1, \ldots, p$. This is impossible in view of the assumption that there exist $i \neq j$ such that either $\beta_{i}<\beta_{j}$ or $B_{i} \neq B_{j}$.

Proof of Theorem 5.1. Multiplying both the sides (5.3) from the left by $\pi$ we get

$$
\sum_{i=1}^{p} \pi_{i} h_{i} \beta_{i}\left(\hat{B}_{i}(\gamma)-1\right)=\gamma \pi \mathbf{h}
$$

from which we have that the adjustment coefficient for the Markov modulated model is the solution of

$$
\gamma=\sum_{i=1}^{p} \frac{\pi_{i} h_{i} \beta_{i}\left(\hat{B}_{i}(\gamma)-1\right)}{\pi \mathbf{h}}
$$

By Lemmas 2.3 and 5.1,

$$
\gamma=\frac{1}{\pi \mathbf{h}} \sum_{i=1}^{p} \pi_{i} \beta_{i} h_{i}\left(\hat{B}_{i}(\gamma)-1\right)>\sum_{i=1}^{p} \pi_{i} \beta_{i}\left(\hat{B}_{i}(\gamma)-1\right),
$$

from which we get (5.6).

\section{PERTURBATION ANALYSIS FOR THE ADJUSTMENT COEFFICIENT}

In this section we assume, similar as in Example 3.2, that the intensity matrix for the Markov environment is parameterized as follows: $\Lambda(\varepsilon)=\Lambda_{0} / \varepsilon$, whereas the $\beta_{i}$ and $B_{i}$ are fixed. The corresponding adjustment coefficient is denoted by $\gamma(\varepsilon)$. Thus $\gamma(\varepsilon) \rightarrow \gamma^{*}$ as $\varepsilon \downarrow 0$, and our aim is to compute the sensitivity of $\gamma(\varepsilon)$ at $\varepsilon=0+0$, i.e. the right derivative

$$
\frac{d^{+} \gamma}{d \varepsilon}(0)
$$

A dual result deals with the limit $\varepsilon \rightarrow \infty$. Here we put $a=1 / \varepsilon$, note that $\gamma(a) \rightarrow \min _{i=1, \ldots, p} \gamma_{i}$ and compute

$$
\frac{d^{+} \gamma}{d a}(0)
$$

The basic equation is again (5.3) where $\boldsymbol{\Lambda}, \gamma, \boldsymbol{h}$ depend on the parameter ( $\varepsilon$ or $a$ ).

The following result quantifies the effect on the adjustment coefficient of adding a small but rapid Markov-modulation to the compound Poisson model. Similarly, Proposition 6.2 below deals with a small but slow Markov-modulation.

Proposition 6.1 In the case $\varepsilon \downarrow 0$ we have

$$
\frac{d^{+} \gamma}{d \varepsilon}(0)=\frac{1}{\pi K^{\prime}\left(\gamma^{*}\right) e} \pi \boldsymbol{K}\left(\gamma^{*}\right)\left(\boldsymbol{\Lambda}_{0}-\boldsymbol{e} \boldsymbol{\pi}\right)^{-1} \boldsymbol{K}\left(\gamma^{*}\right) \boldsymbol{e}
$$

Proof. The existence of the right derivative follows by the implicit function theorem and $\operatorname{det}(\boldsymbol{\Lambda}+\boldsymbol{\varepsilon} \boldsymbol{K}(\gamma(\varepsilon)))=0$. Multiplying (5.3) by $\varepsilon$, we obtain

$$
\mathbf{0}=\left(\Lambda_{0}+\varepsilon \boldsymbol{K}(\gamma)\right) \boldsymbol{h}
$$


Differentiation with respect to $\varepsilon$ gives

$$
\mathbf{0}=\left(\boldsymbol{K}(\gamma)+\varepsilon \gamma^{\prime} \boldsymbol{K}^{\prime}(\gamma)\right) \boldsymbol{h}+\left(\boldsymbol{\Lambda}_{0}+\varepsilon \boldsymbol{K}(\gamma)\right) \boldsymbol{h}^{\prime} .
$$

Normalizing $\boldsymbol{h}$ by $\pi \boldsymbol{h}=1$, we have $\boldsymbol{\pi} \boldsymbol{h}^{\prime}=0, \boldsymbol{h}(0)=\boldsymbol{e}$. Hence letting $\varepsilon \downarrow 0$ in (6.2) yields

$$
\mathbf{0}=\boldsymbol{K}\left(\gamma^{*}\right) \boldsymbol{e}+\boldsymbol{\Lambda}_{0} \boldsymbol{h}^{\prime}(0+0)=\boldsymbol{K}\left(\gamma^{*}\right) \boldsymbol{e}+\left(\boldsymbol{\Lambda}_{0}-\boldsymbol{e} \pi\right) \boldsymbol{h}^{\prime}(0+0),
$$

i.e.

$$
h^{\prime}(0+0)=-\left(\Lambda_{0}-e \pi\right)^{-1} K\left(\gamma^{*}\right) e
$$

Differentiating (6.2) once more and letting $\varepsilon \downarrow 0$ we get

$$
\mathbf{0}=2 \gamma^{\prime}(0+0) \boldsymbol{K}^{\prime}\left(\gamma^{*}\right) \boldsymbol{e}+2 \boldsymbol{K}\left(\boldsymbol{\gamma}^{*}\right) \boldsymbol{h}^{\prime}(0+0)+\boldsymbol{\Lambda}_{0} \mathbf{h}^{\prime \prime}(0+0) \text {. }
$$

Multiplying (6.4) by $\pi$ to the left we get

$$
\mathbf{0}=2 \gamma^{\prime}(0+0) \pi K^{\prime}\left(\gamma^{*}\right) e+2 \pi \boldsymbol{K}\left(\gamma^{*}\right) h^{\prime}(0+0) .
$$

Inserting (6.3) yields (6.1).

Now turn to the case of $a$. We assume that $0<\gamma_{1}<\gamma_{i}$ for $i=2, \ldots, p$. Then $\gamma \rightarrow \gamma_{1}$ as a $\downarrow 0$ and we may take $\boldsymbol{h}(0)=\boldsymbol{e}_{1}$ (the first unit vector). We get

$$
\mathbf{0}=\left(a \Lambda_{0}+K[\gamma]\right) \boldsymbol{h} .
$$

Differentiation with respect to $a$ gives

$$
\mathbf{0}=\left(\boldsymbol{\Lambda}_{0}+\gamma^{\prime} \boldsymbol{K}^{\prime}(\gamma)\right) \boldsymbol{h}+\left(a \boldsymbol{\Lambda}_{0}+\boldsymbol{K}(\gamma)\right) \boldsymbol{h}^{\prime} .
$$

Letting a $\downarrow 0$ in (6.6) and multiplying by $e_{1}$ to the left we get $0=\lambda_{11}+\gamma^{\prime}(0) \kappa_{1}^{\prime}\left(\gamma_{1}\right)$ +0 (using $\kappa_{1}(\gamma(0))=0$ to infer that the first component of $\boldsymbol{K}(\gamma(0)) \boldsymbol{h}^{\prime}(0+0)$ is 0 ), and we have proved:

Proposition 6.2 If $0<\gamma_{1}<\gamma_{i}$ for $i=2, \ldots, p$, then $\frac{d^{+} \gamma}{d a}(0)=-\frac{\lambda_{11}}{\kappa_{1}^{\prime}\left(\gamma_{1}\right)}$.

\section{REFERENCES}

[1] S. Asmussen (1989) Risk theory in a Markovian environment. Scand. Actuarial J., 69-100.

[2] S. Asmussen, L. Fløe Henriksen and C. Klüppelberg (1994) Large claims approximations for risk processes in a Markovian environment. Stoch. Proc. Appl. 54, 29-43.

[3] S. ASMUSSEN and T. RoLSKI (1991). Computational methods in risk theory: A matrix-algorithmic approach. Insurance: Mathematics and Economics 10, 259-274.

[4] S. Asmussen and T. RoLSKI (1994) Risk theory in a periodic environment; the Cramér-Lundberg approximation and Lundberg's inequality. Math. Oper. Res., 19, 410-433.

[5] S. AsMuSSEN and V. SchMidT (1995) Ladder height distributions with marks. Stoch. Proc. Appl. (to appear).

[6] S. Asmussen (1994) Ruin Probabilities. Book manuscript, Aalborg University. To be published by World Science Publishers, Singapore.

[7] C. Chang, X.L. Chao and M. Pinedo (1991) Monotonicity results for queues with doubly stochastic Poisson arrivals: Ross's conjecture. Adv. Appl. Prob. 23, 210-228. 
[8] C. Chang and R. Nelson (1993) Perturbation analysis of the M/M/1 queue in a Markovian environment via the matrix-geometric method. Stochastic Models 9, 233-246.

[9] D.J. DALEY and T. RoLSKI (1984) Some comparability results for waiting times in single- and manyserver queues. J. Appl. Prob. 21, 887-900.

[10] J.D. Esary, F. Proschan and D.W. WALKup (1967) Association of random variables, with applications. Ann. Math. Statist. 38, 1466-1474.

[11] P. Franken, D. KönIG, U. ARndt and V. Schmidt (1982) Queues and Point Processes. J. Wiley \& Sons, Chichester.

[12] M.J. Goovaerts, R. KaAs, A.E. van HeErwarden and T. Bauwelinckx (1990) Effective Actuarial Methods. North-Holland, Amsterdam.

[13] B. GrigeLIONIS (1993) Two-sided Lundberg inequalities in a Markovian environment. Liet. Matem. Rinkinys 33.

[14] A.E. Van HeERWARden (1991) Ordering of Risks: Theory and Actuarial Applications. Tinbergen Institute Research Series 20, Amsterdam.

[15] J. JANSSEN (1980) Some transient results on the M/SM/1 special semi-Markov model in risk and queueing theories. Astin Bulletin 11, 41-51.

[16] J. JANSSEn and J.M. Reinhard (1985) Probabilities de ruine pour une classe de modeles de risque semi-Markoviens. Astin Bulletin 15, 123-133.

[17] F.P. KELLY (1979) Reversibility and Stochastic Networks. J. Wiley \& Sons, Chichester.

[18] C. KLUPPELBERG (1993) Asymptotic ordering of risks and ruin probabilities. Insurance: Mathematics and Economics 12, 259-264.

[19] D. KonlG and V. SCHMIDT (1992) Random Point Processes. Teubner, Stuttgart (in Germ.).

[20] A.M. MAKOWSKI (1994) On an elementary characterization of the increasing convex ordering, with an application. J. Appl. Prob. 31, 834-840.

[21] D.S. Mitrinović, J.E. PeĆARIĆ and A.M. FInk (1993) Classical and Neuv Inequalities in Analysis. Kluwer Academic Publishers, Amsterdam.

[22] M.F. NeUts (1978) The $M / M / 1$ queue with randomly varying arrival rate and service rates. Opsearch, 155, 139-157

[23] M.F. Neuts (1981) Matrix-Geometric Solutions in Stochastic Models. The John Hopkins University Press. Baltimore.

[24] J.M. REINHARD (1984). On a class of semi-Markov risk models obtained as classical risk models in a markovian environment. Astin Bulletin 14, 23-43.

[25] T. RoLSKI (1981) Queues with non-stationary input stream: Ross's conjecture. Adv. Appl. Prob. 13, 603-618.

[26] T. RoLSKI (1989) Queues with nonstationary inputs. Queueing Systems 15, 113-129.

[27] S.M. Ross (1978) Average delay in queues with non-stationary Poisson arrivals. J. Appl. Prob. 8, 814-827.

[28] D. STOYAN (1983) Comparison Methods for Queues and Other Stochastic Models. J. Wiley \& Sons, Chichester.

Søren Asmussen

Institute of Electronic Systems, Aalborg University

Fr. Bajersv. 7, DK-9220 Aalborg, Denmark

ANDREAS FREY

Department of Stochastics, University of Ulm

Helmholtzstr. 18, D-89069 Ulm, Germany

TOMASZ ROLSKI*

Mathematical Institute, University of Wroclaw

pl. Grunwaldzki 2/4, 50-384 Wroclaw, Poland

VOLKER SCHMIDT

Department of Stochastics, University of Ulm

Helmholtzstr. 18, D-89069 Ulm, Germany

* Supported in part by KBN under grant $640 / 2 / 9$ 